\title{
Sensitivity of doxorubicin-resistant cells to sorafenib: Possible role for inhibition of eukaryotic initiation factor-2 $\alpha$ phosphorylation
}

\author{
MASAKI SHIOTA $^{1}$, MASATOSHI ETO ${ }^{2}$, AKIRA YOKOMIZO ${ }^{1}$, YASUHIRO TADA ${ }^{1}$, ARIO TAKEUCHI ${ }^{1}$, \\ MOMOE ITSUMI $^{1}$, KATSUNORI TATSUGAMI ${ }^{1}$, TAKESHI UCHIUMI ${ }^{3}$ and SEIJI NAITO $^{1}$ \\ ${ }^{1}$ Department of Urology and ${ }^{3}$ Department of Clinical Chemistry and Laboratory Medicine, \\ Graduate School of Medical Sciences, Kyushu University, Fukuoka; ${ }^{2}$ Department of Urology, \\ Graduated School of Medicine, Kumamoto University, Kumamoto, Japan
}

Received April 7, 2010; Accepted May 26, 2010

DOI: 10.3892/ijo_00000700

\begin{abstract}
Patients with advanced cancer including breast cancer, hepatocellular cancer and urothelial cancer frequently receive a chemotherapy regimen containing doxorubicin. However, doxorubicin-resistance is a major obstacle for cancer chemotherapy. Recently, several molecular-targeted agents have become available. Sorafenib (BAY 43-9006) is known to target multiple kinases and has demonstrated activity in renal cell and hepatocellular cancer. In this study, sorafenib was found to inhibit phosphorylation of the eukaryotic initiation factor- $2 \alpha(\mathrm{eIF} 2 \alpha)$, induce cell cycle arrest at G2 phase and increase cellular apoptosis in doxorubicinresistant human urothelial cell lines. An eIF $2 \alpha$ kinase, PERK was responsible for eIF $2 \alpha$ phosphorylation and PERK knockdown induced cellular apoptosis similar to sorafenib treatment in doxorubicin-resistant cancer cells. Furthermore, sorafenib sensitized doxorubicin-resistant cancer cells, but not their parental cells to oxidative stress exerted by both hydrogen peroxide and doxorubicin. In addition, PERK knockdown sensitized doxorubicin-resistant cancer cells to oxidative stress. In conclusion, PERK inhibition using sorafenib with or without doxorubicin might be a promising therapeutic approach for doxorubicin-resistant cancers retaining high phosphorylation levels of eIF $2 \alpha$.
\end{abstract}

\section{Introduction}

The majority of cancer patients with advanced stage disease receive chemotherapy. Although anticancer agents decrease patient tumor burdens and expand life expectancy, nonresponsiveness or development of chemotherapy resistance are major obstacles for effective cancer treatment. We have previously investigated mechanisms of resistance to anti-

Correspondence to: Dr Seiji Naito, Department of Urology, Graduate School of Medical Sciences, Kyushu University, 3-1-1 Maidashi, Higashi-ku, Fukuoka 812-8252, Japan

E-mail: naito@uro.med.kyushu-u.ac.jp

Key words: doxorubicin, eIF2 $\alpha$, PERK, sorafenib, urothelial cancer cancer drugs including cisplatin, vincristine, etoposide and doxorubicin (1-11). Doxorubicin has been administered for many types of solid tumors including breast cancer, hepatocellular cancer and urothelial cancer. Several molecules that are associated with the acquisition of doxorubicin resistance have been identified, including detoxifying enzymes, drugefflux pumps and apoptosis-related genes $(12,13)$. We have previously established doxorubicin-resistant cancer cells and shown that these cells expressed an increased multidrugresistance 1 (MDR1) and MDR-related protein (MRP) mRNA and a decreased level of topoisomerase II mRNA (4). In addition, using 47 clinical samples of bladder cancer, we confirmed the significant correlation of MDR1, MRP1 and MRP3 mRNA levels with resistance to doxorubicin, and showed that the expression of MDR1, MRP1, MRP2 and MRP3 in recurrent tumors and residual tumors after chemotherapeutic treatment was higher than that in untreated primary tumors (6). However, overcoming doxorubicin resistance remains unresolved.

Sorafenib (BAY 43-9006) is a novel bi-aryl urea that has been previously shown to inhibit Raf-1 and tumor cell line proliferation and tumor xenograft models $(14,15)$. In addition to Raf-1 inhibition, sorafenib is a multikinase inhibitor, which targets vascular endothelial growth factor receptor (VEGFR) 2 and 3; platelet-derived growth factor receptor- $\beta$ (PDGFR- 3 ); FMS-like tyrosine kinase 3 (FLT-3); c-KIT; and RET-receptor tyrosine kinase $(16,17)$. The clinical benefit of sorafenib was found in a phase II randomized trial, which indicated a lengthened progression-free survival with sorafenib compared with placebo in patients with metastatic renal cell cancer (RCC) (18). In addition, a subsequent phase III randomized controlled trial [treatment approaches in renal cancer global evaluation trial (TARGET)] confirmed the findings of the phase II trial (19). We have previously conducted phase II study to investigate the efficacy, safety, and pharmacokinetics of sorafenib in Japanese patients with advanced renal cell carcinoma, and revealed that sorafenib exerted encouraging efficacy and was well tolerated in Japanese patients with metastatic RCC (20).

Phosphorylation of the $\alpha$ subunit of eukaryotic initiation factor-2 (eIF $2 \alpha$ ) plays an essential role in regulating protein synthesis in response to a diverse range of environmental 
stresses (21-23). Four eIF2 $\alpha$ kinases have been identified in mammals, each responding to different stresses through their unique regulatory regions. For example, phosphorylation of eIF2 $\alpha$ by PERK (PKR-like ER kinase; also known as EIF2AK3) is induced by accumulation of malfolded proteins in the endoplasmic reticulum (ER) (24-26). Phosphorylation of eIF $2 \alpha$ during ER stress inhibits global translation by lessening eIF2-GTP (21-23). Despite an overall reduction in protein synthesis, eIF $2 \alpha$ phosphorylation increases the preferential translation of ATF4 mRNA, encoding a basic zipper transcription activator that is important for directing the expression of genes involved in metabolism, the redox status of cells, apoptosis and drug resistance (27-30). Decreased protein synthesis conserves energy and provides sufficient time for ATF4 and other stress-responsive transcription factors, to reconfigure gene expression that would block or ameliorate damage elicited by the underlying stress. Other members of the eIF2 $\alpha$ kinase family include GCN2 (EIF2AK4), whose activity is enhanced by amino acid depletion, UV irradiation or proteasome inhibition; PKR (EIF2AK2), which function in an antiviral pathway; and HRI (EIF2AK1), which is regulated by heme deficiency and oxidative stress $(22,23,26,31)$. Aberrations in these eIF $2 \alpha$ kinase pathways are associated with a number of diseases, including diabetes, viral infection, anemia, neurological disorders and various cancers.

In this study, we investigated whether multikinase inhibitor, sorafenib could suppress the growth of doxorubicinresistant cancer cells. Then, we attempted to elucidate a mechanism by which sorafenib inhibited the growth of doxorubicin-resistant cancer cells. Furthermore, we investigated the effects of sorafenib administration and PERK inhibition combined with hydrogen peroxide and doxorubicin.

\section{Materials and methods}

Cell culture and chemical. Human urothelial carcinoma KK47 cells, T24 cells and their doxorubicin-resistant cells were cultured in Eagle's minimal essential medium. Doxorubicinresistant KK47/ADR and T24/ADR were established and maintained as described previously (4). Media were purchased from Invitrogen (San Diego, CA, USA) and contained 10\% fetal bovine serum. Cell lines were maintained in a $5 \% \mathrm{CO}_{2}$ atmo-sphere at $37^{\circ} \mathrm{C}$. Sorafenib was obtained from Bayer Pharma-ceuticals Corporation (West Haven, CT, USA). For assay, stock solution was prepared in dimethyl sulfoxide (DMSO) $(10 \mathrm{mM})$, and stored at $-20^{\circ} \mathrm{C}$ until use. Stock solution was diluted to appropriate concentration in culture medium before addition to the cells.

Antibodies. Antibodies against GCN2 (no. 3302), p-eIF2 $\alpha$ (no. 9721), eIF $2 \alpha$ (no. 2103) and p-ERK1/2 (no. 9106) were purchased from Cell Signaling Technology (Danvers, MA, USA). Antibody against ERK1 (sc-94), PARP (sc-1561) and PERK (sc-13073) were purchased from Santa Cruz Biotechnology (Santa Cruz, CA, USA). Anti-PKR (no. 1511-1) antibody was purchased from Epitomics (Burlingame, CA, USA). Anti-lamin B 1 and anti- $B$-actin antibodies were purchased from Sigma, USA. Anti-cleaved-PARP antibody was purchased from Promega (Madison, WI, USA).
Knockdown analysis using siRNAs. Knockdown analysis using siRNAs was performed as described previously $(10,11,30,31)$. Briefly, the following double-stranded RNA oligonucleotides were commercially generated: 5'-GGUA AAAAGCAGUGGGAUUTT-3' (sense) and 5'-AAUCCCAC UGCUUUUUACCTT-3' (antisense) for PERK no. 1 (B-Bridge International, Mountain View, CA, USA); 5'-GGGAAAAGG UAAUGCGAGATT-3' (sense) and 5'-UCUCGCAUUAC CUUUUCCCTT-3' (antisense) for PERK no. 2 (B-Bridge International); 5'-CCACAUGAUAGGAGGUUUATT-3' (sense) and 5'-UAAACCUCCUAUCAUGUGGTT-3' (antisense) for PKR (B-Bridge International); 5'-AUUAGAU GCACUCAGGACCUUAUGC-3' (sense) and 5'-GCAUA AGGUCCUGAGUGCAUCUAAU-3' (antisense) for GCN2 (Invitrogen). T24, K47 and their doxorubicin-resistant cells were transfected with $40 \mathrm{nM}$ of the indicated siRNA using Lipofectamine 2000 (Invitrogen) according to the manufacturer's instruction.

Cytotoxicity analysis. Cytotoxicity analysis was performed as described previously $(10,31)$. Briefly, T24, KK47 and their doxorubicin-resistant cells $\left(2.5 \times 10^{3}\right)$ applied to $0.5 \mu \mathrm{M}$ of sorafenib or transfected with $40 \mathrm{nM}$ of the indicated siRNA were seeded into 96-well plates. The following day, the indicated concentrations of hydrogen peroxide or doxorubicin and/or N-acetyl-L-cysteine (NAC) were applied. After $48 \mathrm{~h}$, surviving cells were stained with alamarBlue Assay (TREK Diagnostic systems, Cleaveland, OH, USA) for $180 \mathrm{~min}$ at $37^{\circ} \mathrm{C}$. The absorbance was then measured using a plate reader $\left(\mathrm{ARVO}^{\mathrm{TM}} \mathrm{MX}\right.$, Perkin-Elmer Inc., Waltham, MA, USA).

Flow cytometry analysis. Flow cytometry analysis was performed as described previously $(10,31)$. Briefly, T24, KK47 and their doxorubicin-resistant cells $\left(2.0 \times 10^{5}\right)$ were seeded in 6-well plates, applied to $2.5 \mu \mathrm{M}$ of sorafenib and cultured for $24 \mathrm{~h}$, or transfected with $40 \mathrm{nM}$ of the indicated siRNA and cultured for $72 \mathrm{~h}$. The cells were harvested, washed twice with ice-cold PBS containing $0.1 \%$ bovine serum albumin (BSA) and resuspended in $70 \%$ ethanol. After washing twice with ice-cold PBS, cells were resuspended in PBS containing $0.1 \%$ BSA, incubated with RNase (Roche Applied Science, Indianapolis, IN, USA) and stained with propidium iodide (Sigma). Cells were analyzed using a FACSCalibur (BD Biosciences, San Jose, CA, USA).

Western blotting. Whole-cell extracts were prepared with phosphatase inhibitor Cocktail (Nacalai Tesque, Kyoto, Japan) as previously described $(10,11,30,31)$. The protein concentration was determined using a Protein Assay Kit (Bio-Rad, Hercules, CA, USA), based on Bradford's method. Whole-cell extracts $(30 \mu \mathrm{g})$ were separated by sodium dodecyl sulfate-polyacrylamide gel electrophoresis (SDSPAGE) and transferred to polyvinylidene difluoride microporous membranes (GE Healthcare Bio-Science, Piscataway, NJ, USA) using a semi-dry blotter. The blotted membranes were incubated for $1 \mathrm{~h}$ at room temperature with a primary antibody. Membranes were then incubated for $40 \mathrm{~min}$ at room temperature with a peroxidase-conjugated secondary antibody. The bound antibody was visualized using an ECL 
A

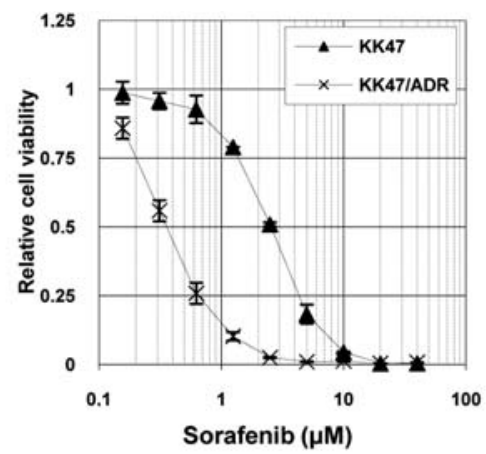

B

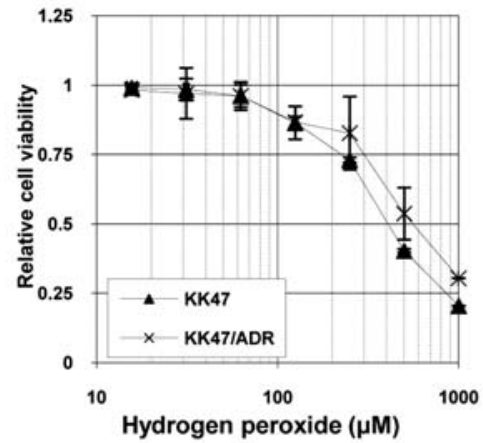

C

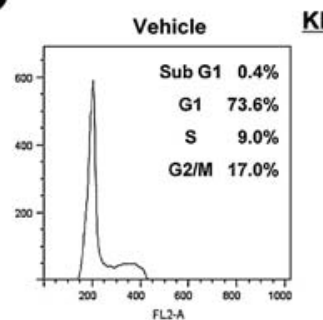

KK47 Sorafenib $2.5 \mu \mathrm{M}$

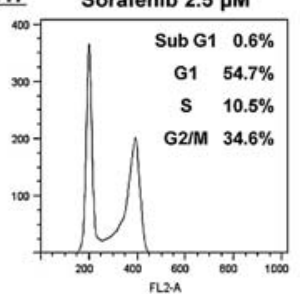

Vehicle

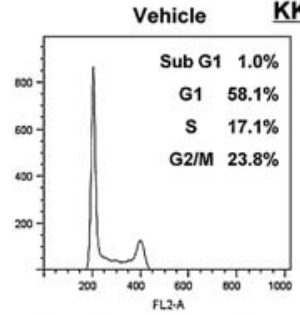

KK47/ADR Sorafenib $2.5 \mu \mathrm{M}$

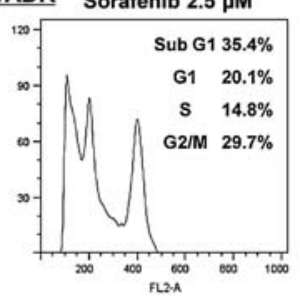

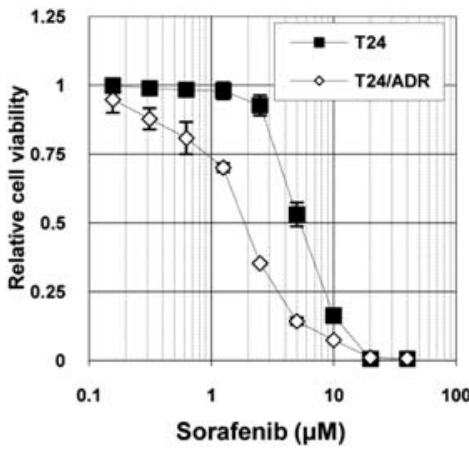

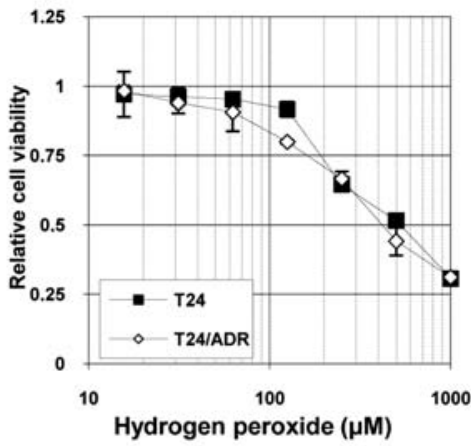

D

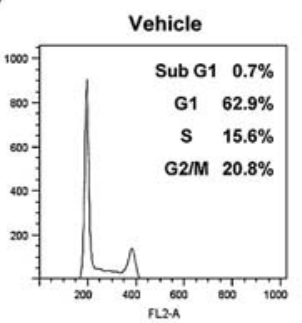

T24 Sorafenib $2.5 \mu \mathrm{M}$

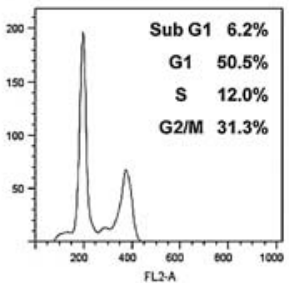

T24/ADR Sorafenib $2.5 \mu \mathrm{M}$
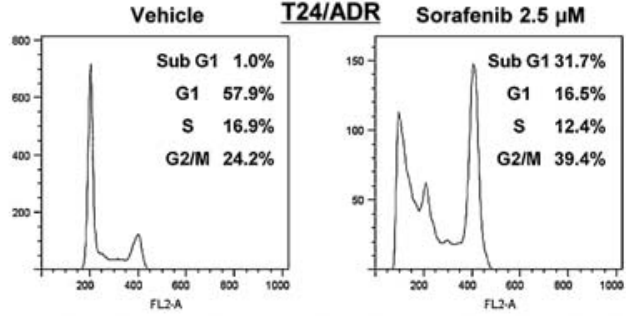

Figure 1. Sorafenib, but not hydrogen peroxide suppresses survival of doxorubicin-resistant cancer cells more effectively than their parental cells. (A) and (B) KK47, T24 and their doxorubicin-resistant cells were seeded into 96-well plates. The following day, various concentrations of sorafenib (A) or hydrogen peroxide (B) were applied. After $48 \mathrm{~h}$, cell survival was analyzed by cytotoxicity assay. Cell survival in the absence of sorafenib or hydrogen peroxide corresponds to 1 . All values are representative of at least three independent experiments. Boxes, mean; bars, \pm s.d. (C) KK47, T24 and their doxorubicinresistant cells were applied to vehicle or $2.5 \mu \mathrm{M}$ of sorafenib for $24 \mathrm{~h}$, and the cells were stained with propidium iodide and analyzed by flow cytometry. The cell cycle fractions are shown at the top right of each graph.

kit (GE Healthcare Bio-Science) and membranes were exposed to Kodak X-OMAT film.

\section{Results}

Sorafenib, but not hydrogen peroxide suppresses doxorubicinresistant cancer cell survival more effectively than their parental cells. We have been investigating mechanisms of a resistance to anticancer drug and aiming to overcome anticancer-drug resistance (1-11). We previously established doxorubicin-resistant cells of urothelial cancer (4). To examine which anticancer agents are effective for doxorubicinresistant cancer cells, we carried out cytotoxicity assays to compare drug sensitivity between doxorubicin-resistant cancer cells and their parental cells using various anticancer drugs. First, doxorubicin-resistant KK47/ADR cells and their parental KK47 cells were subjected to cytotoxicity assay using sorafenib. Although doxorubicin-resistant cells were cross-resistant to various anticancer agents as previously reported (4), to our surprise, the results showed that sorafenib suppressed survival of KK47/ADR cells more effectively than KK47 cells (Fig. 1A, left panel). To examine whether this finding is applicable to other cell lines, we investigated the cytotoxicity of sorafenib using T24 cells and their doxorubicin-resistant T24/ADR cells. Sorafenib repeatedly suppressed survival of doxorubicin-resistant T24-cells more 
A

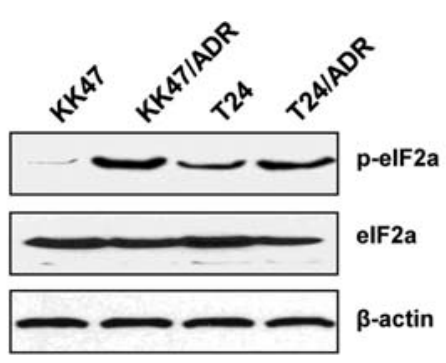

B

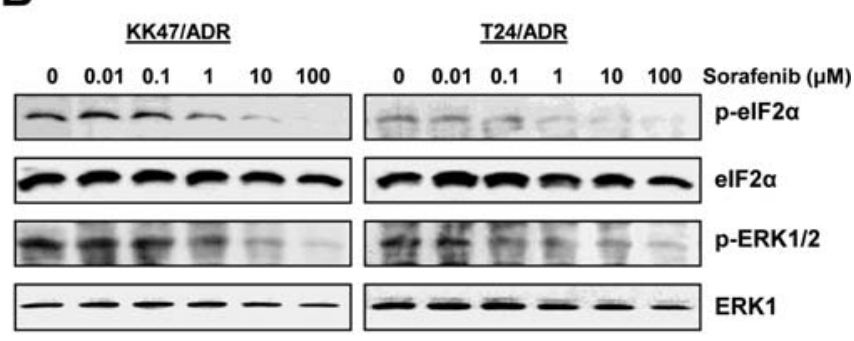

C

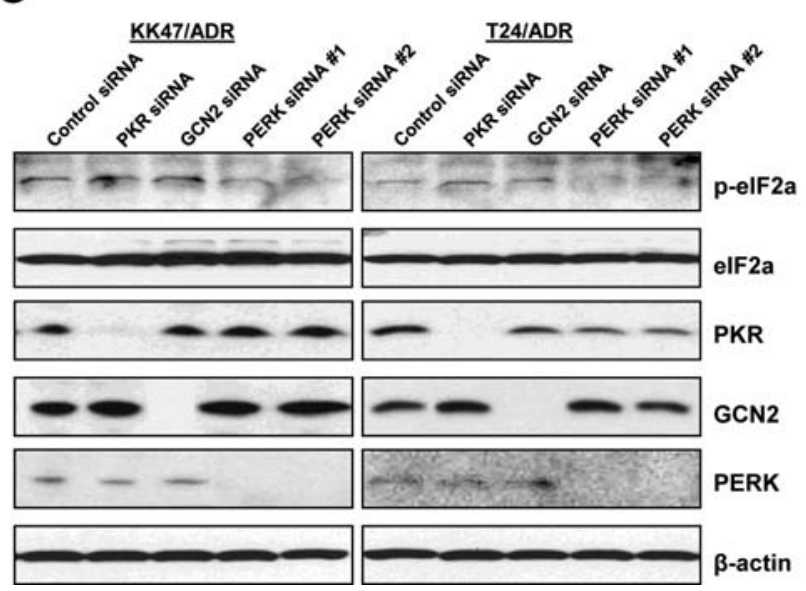

Figure 2. Sorafenib inhibits eIF2 $\alpha$ phosphorylation PERK-dependently with higher level in doxorubicin-resistant cells. (A) Whole-cell extracts of KK47, T24 and their doxorubicin-resistant cells were subjected to SDS-PAGE, and Western blotting was performed using the indicated antibodies. (B) KK47/ADR and T24/ADR cells were cultured with various concentrations of sorafenib for $6 \mathrm{~h}$, and the cells were harvested. Whole-cell extracts were subjected to SDS-PAGE, and Western blotting was performed using the indicated antibodies. (C) KK47/ADR and T24/ADR cells were transfected with $40 \mathrm{nM}$ of control siRNA, PKR siRNA, GCN2 siRNA, PERK siRNA no. 1 or PERK siRNA no. 2. At $72 \mathrm{~h}$ after transfection, whole-cell extracts were subjected to SDS-PAGE, and Western blotting was performed using the indicated antibodies.

effectively than their parental cells (Fig. 1A, right panel). On the other hand, cytotoxicity to hydrogen peroxide did not differ between doxorubicin-resistant cells and their parental cells (Fig. 1B). To confirm these findings and investigate the mechanism of sorafenib cytotoxicity, flow cytometry analysis was carried out. When KK47 cells were treated with $2.5 \mu \mathrm{M}$ of sorafenib, KK47 cells were arrested at G2 phase of cell cycle, but showed no cell death as indicated by no increase in the sub-G1 fraction of cell cycle analysis. In contrast, KK47/ ADR cells were arrested at G2 phase of cell cycle and showed a marked increase in the sub-G1 fraction when treated with
$2.5 \mu \mathrm{M}$ of sorafenib which indicates cell death. Similarly, when T24 and T24/ADR cells were treated with sorafenib, T24/ADR cells were arrested at G2 phase of cell cycle and showed cell death more clearly than T24 cells (Fig. 1C).

Sorafenib inhibits eIF2a phosphorylation PERK-dependently with higher level in doxorubicin-resistant cells. To elucidate a possible mechanism of higher sensitivity of doxorubicinresistant cells to the multikinase inhibitor sorafenib, we compared phosphorylation status of sorafenib targets between doxorubicin-resistant cells and their parental cells. Basal phosphorylation status of known targets of sorafenib such as VEGFR2 and ERK did not differ between doxorubicin-resistant cells and their parental cells (data not shown). However, phosphorylated form of eIF2 $\alpha$ which we have found to be a target of sorafenib in RCC cells (32) was higher in both doxorubicin-resistant cell lines (KK47/ADR and T24/ADR cells) compared to their parental cells (Fig. 2A). We investigated whether sorafenib could inhibit phosphorylation of eIF $2 \alpha$ as was shown previously in RCC cells. Expectedly, phosphorylated form of ERK1/2 as well as eIF $2 \alpha$ was reduced by sorafenib as shown in Fig. $2 \mathrm{~B}$. Sorafenib concentration of $1 \mu \mathrm{M}$ inhibited eIF $2 \alpha$ phosphorylation to $50 \%$ of vehicle treatment in both doxorubicinresistant cell lines. There are four members of eIF2 $\alpha$ kinases; HRI, PKR, PERK and GCN2. To examine which form is responsible for eIF $2 \alpha$ phosphorylation in doxorubicinresistant urothelial cancer cells, phosphorylation status of eIF $2 \alpha$ was investigated using eIF2 $\alpha$ kinase-specific siRNAs (except for HRI which expression is restricted to erythroblast cells). Silencing of PERK reduced eIF2 $\alpha$ phosphorylation whereas PKR and GCN2 knockdown did not affect phosphorylation of eIF $2 \alpha$ (Fig. 2C).

Silencing of PERK induces cell cycle arrest at G2 phase and cellular apoptosis in doxorubicin-resistant cancer cells similar to sorafenib. Because both sorafenib and PERK inhibition could reduce eIF2 $\alpha$ phosphorylation level (Fig. 2B and $\mathrm{C}$ ), whether PERK knockdown using PERK-specific siRNA could affect cell cycle similar to sorafenib was investigated. Silencing of PERK induced cell cycle arrest at G2 phase and cell death in KK47/ADR cells whereas PERK knockdown induced no effect on cell cycle in KK47 cells (Fig. 3A). Similarly, suppression of PERK induces cell cycle arrest at G2 phase and cell death more effectively in T24/ADR cells than in T24 cells (Fig. 3B). These findings were confirmed by higher increase of cleaved-PARP form indicating cellular apoptosis in doxorubicin-resistant cell lines than their parental cells (Fig. 3C).

Sorafenib sensitizes doxorubicin-resistant urothelial cancer cells to oxidative stress exerted by both hydrogen peroxide and doxorubicin. Because sorafenib could sensitize cancer cells to hydrogen peroxide producing oxidative stress, whether sorafenib could also sensitize doxorubicin-resistant cells to hydrogen peroxide was investigated. As shown in Fig. 4A, sorafenib sensitized both doxorubicin-resistant cell lines to hydrogen peroxide. As expected, addition of NAC abolished the effect of hydrogen peroxide combined with sorafenib in both doxorubicin-resistant cell lines similar to 
A
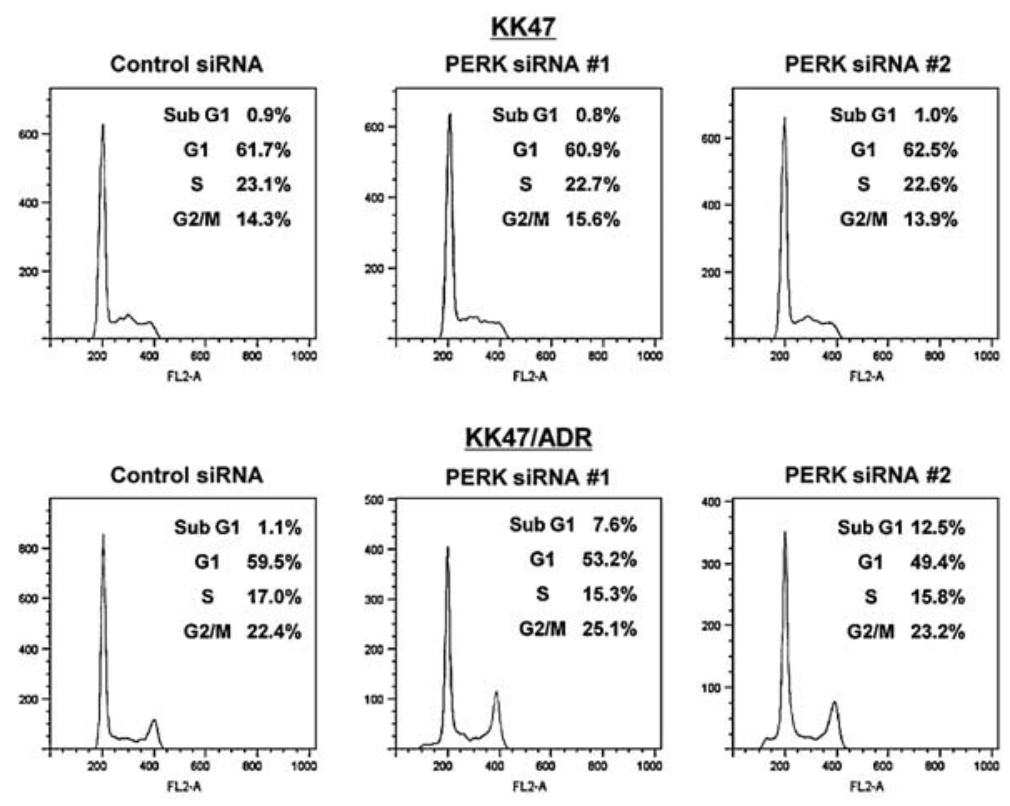

B

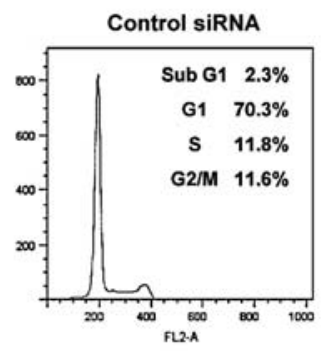

$\underline{T 24}$
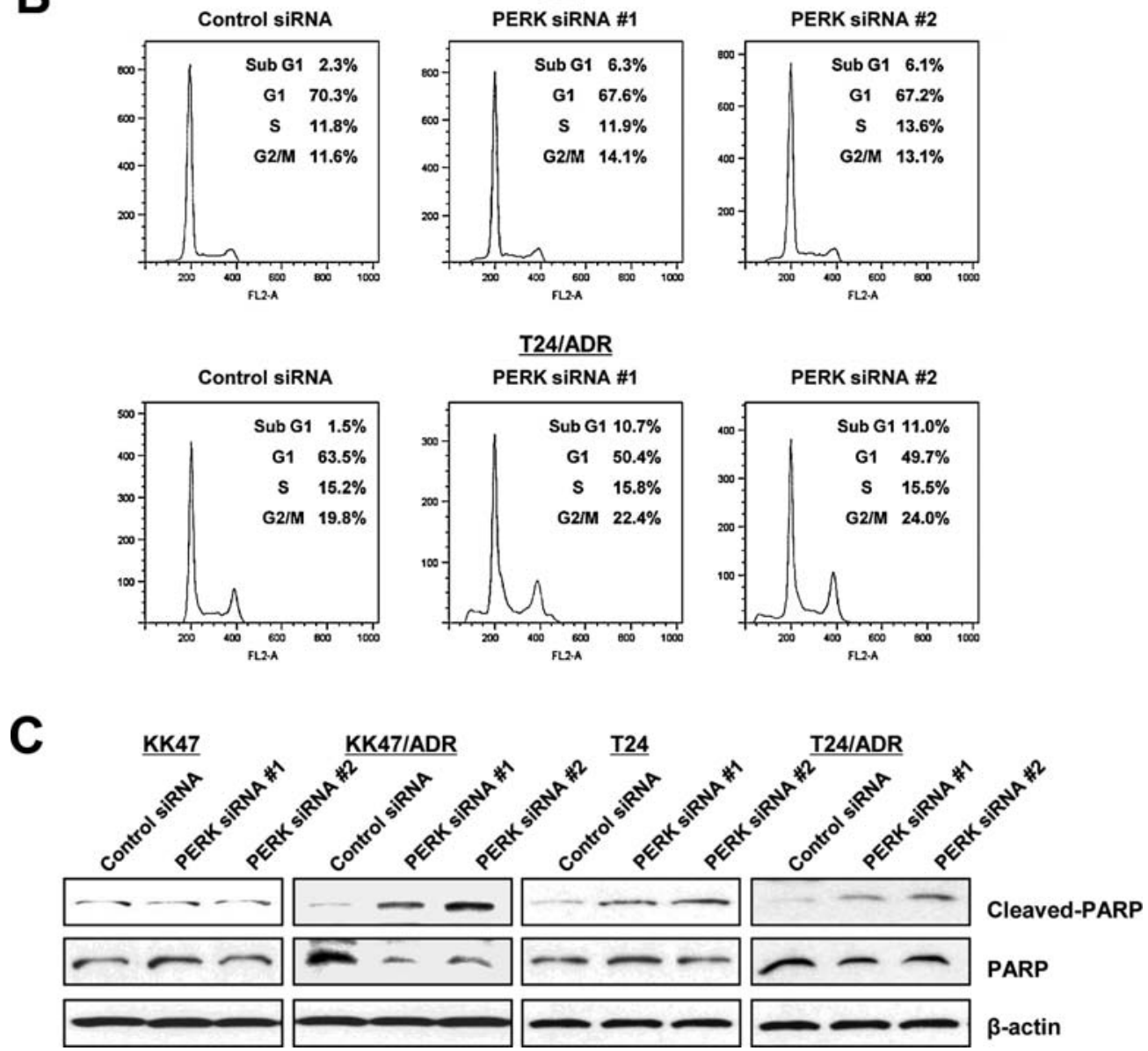

Figure 3. Silencing of PERK induces cell cycle arrest at G2 phase and cell death in doxorubicin-resistant cancer cells similar to sorafenib. (A) and (B) KK47 (A), KK47/ADR (A), T24 (B) and T24/ADR (B) cells were transfected with $40 \mathrm{nM}$ of control siRNA, PERK siRNA no. 1 or PERK siRNA no. 2 . At $72 \mathrm{~h}$ after transfection, cells were stained with propidium iodide and analyzed by flow cytometry. The cell cycle fractions are shown at the top right of each graph. (C) KK47, T24 and their doxorubicin-resistant cells were transfected with $40 \mathrm{nM}$ of control siRNA, PERK siRNA no. 1 or PERK siRNA no. 2 . At $72 \mathrm{~h}$ after transfection, whole-cell extracts were subjected to SDS-PAGE, and Western blotting was performed using the indicated antibodies.

their parental cells (Fig. 4B). Doxorubicin is known to produce high-level oxidative stress to cancer cells (33). Therefore, we assayed cytotoxicity when doxorubicin plus sorafenib were applied. As shown in Fig. 4C, sorafenib sensitized both doxorubicin-resistant cell lines to doxorubicin. However, this favorable effect of doxorubicin plus sorafenib 
A

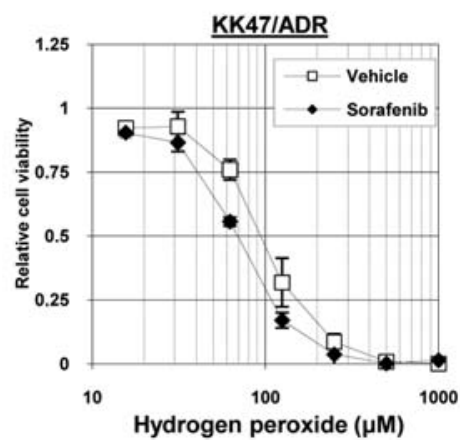

B

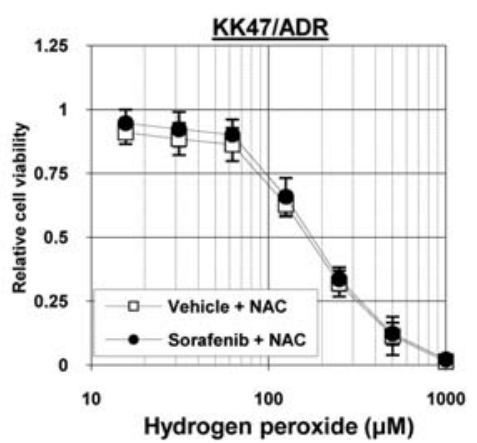

C

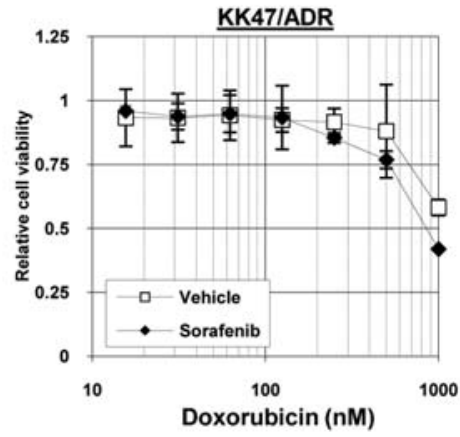

D

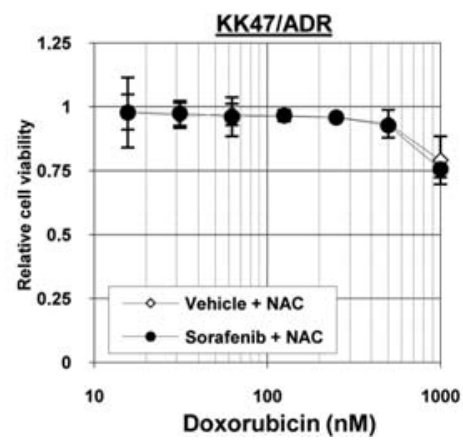

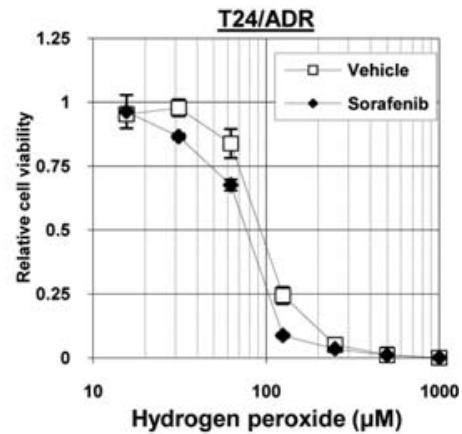
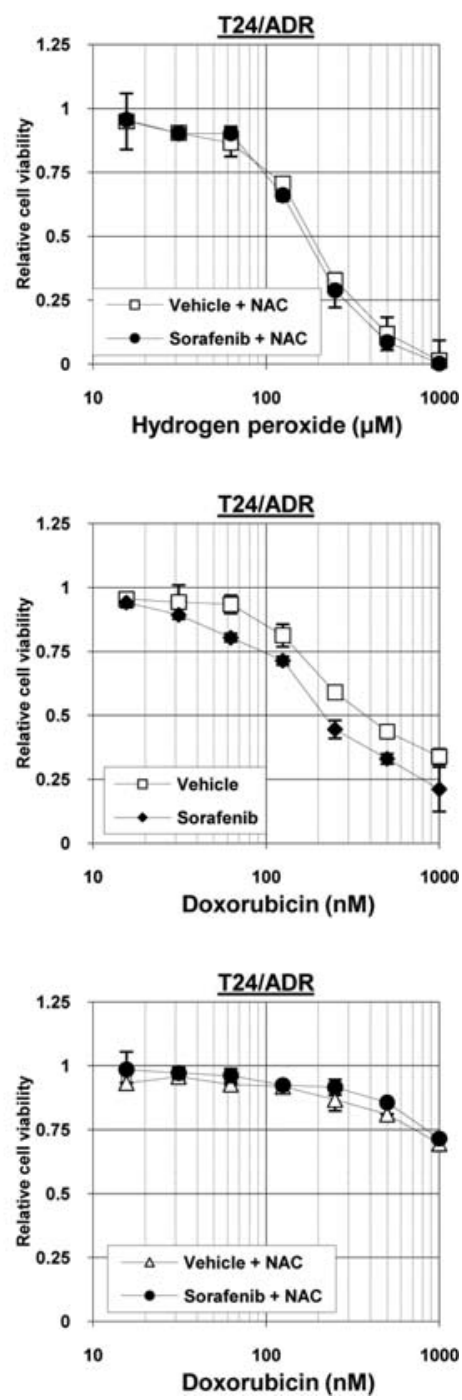

Figure 4. Sorafenib sensitizes doxorubicin-resistant cells to oxidative stress exerted by hydrogen peroxide and doxorubicin. (A) and (C) KK47/ADR and T24/ADR cells applied to $0.5 \mu \mathrm{M}$ of sorafenib were seeded into 96-well plates. The following day, various concentrations of hydrogen peroxide (A) or doxorubicin (C) were applied. After $48 \mathrm{~h}$, cell survival was analyzed by cytotoxicity assay. Cell survival in the absence of hydrogen peroxide corresponds to 1 . All values are representative of at least three independent experiments. Boxes, mean; bars, \pm s.d. (B) and (D) KK47/ADR and T24/ADR cells applied to $0.5 \mu \mathrm{M}$ of sorafenib and $5 \mathrm{mM}$ of NAC were seeded into 96-well plates. The following day, various concentrations of hydrogen peroxide (B) or doxorubicin (D) were applied. Cytotoxicity assay was performed as described in (A) and (C). Boxes, mean; bars, \pm s.d.

was abolished when NAC was added, suggesting that this combinational effect resulted from oxidative stress by doxorubicin (Fig. 4D).

PERK knockdown sensitizes both doxorubicin cells to oxidative stress exerted by hydrogen peroxide and doxorubicin. Because both sorafenib and PERK inhibition reduced eIF $2 \alpha$ phosphorylation level (Fig. 2B and C), PERK knockdown using PERK-specific siRNAs instead of sorafenib was employed for cytotoxicity assay by hydrogen peroxide and doxorubicin. First, cytotoxicity by hydrogen peroxide in doxorubicin-resistant cells transfected with PERK-specific siRNAs was examined. Hydrogen peroxide with PERK knockdown suppressed survival of both doxorubicin-resistant 
A

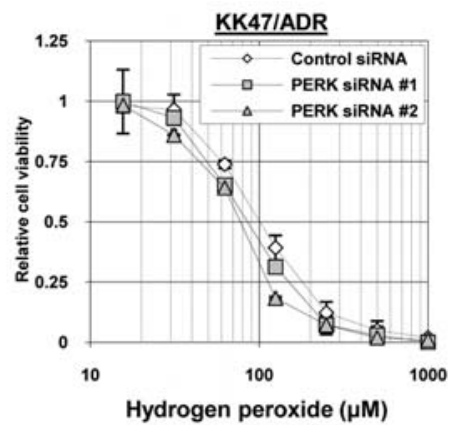

B

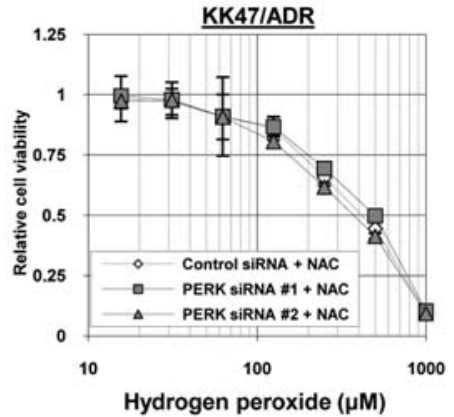

C

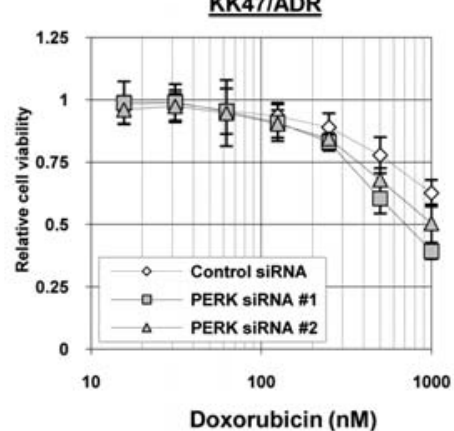

D

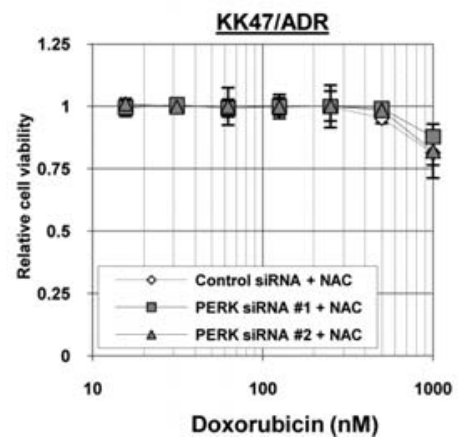

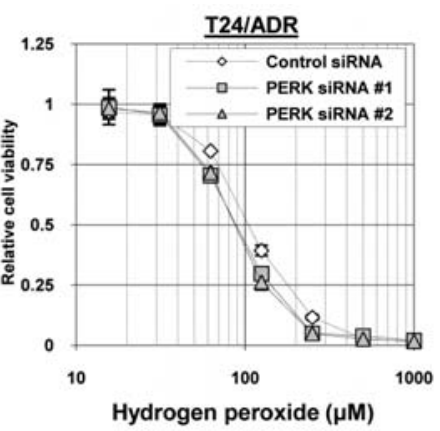

T24/ADR

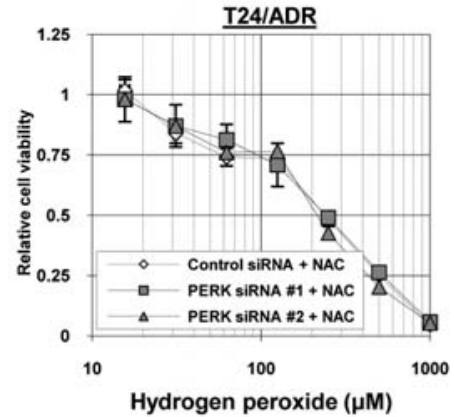

I24/ADR

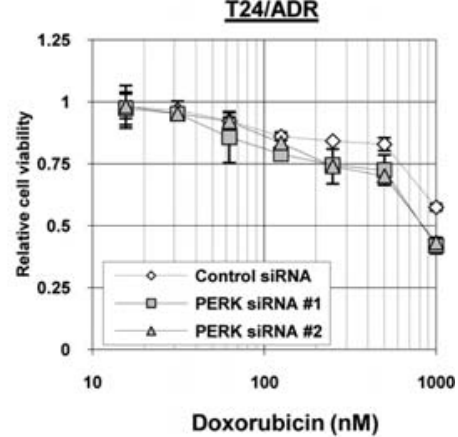

T24/ADR

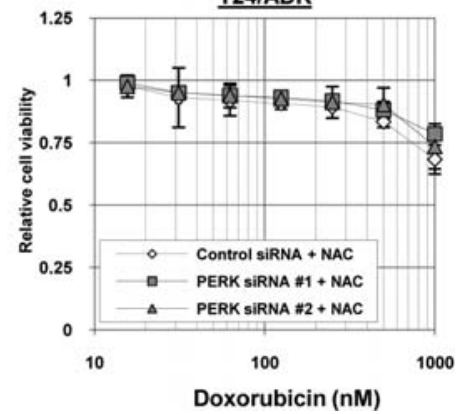

Figure 5. PERK knockdown sensitizes both doxorubicin-resistant cells to oxidative stress exerted by hydrogen peroxide and doxorubicin. (A) and (C) KK47/ADR and T24/ADR cells transfected with $40 \mathrm{nM}$ of control siRNA, PERK siRNA no. 1 or PERK siRNA no. 2 were seeded into 96-well plates. The following day, various concentrations of hydrogen peroxide (A) or doxorubicin (C) were applied. After $48 \mathrm{~h}$, cell survival was analyzed by cytotoxicity assay. Cell survival in the absence of hydrogen peroxide corresponds to 1 . All values are representative of at least three independent experiments. Boxes, mean; bars, \pm s.d. (B) and (D) KK47/ADR and T24/ADR cells transfected with $40 \mathrm{nM}$ of control siRNA, PERK siRNA no. 1 or PERK siRNA no. 2, and applied to 5 mM of NAC were seeded into 96-well plates. The following day, various concentrations of hydrogen peroxide (B) or doxorubicin (D) were applied. Cytotoxicity assay was performed as described in (A) and (C). Boxes, mean; bars, \pm s.d.

cells more effectively compared with hydrogen peroxide alone although an increase of cytotoxicity by sorafenib was modest (Fig. 5A). To confirm a mechanism of an additional effect of PERK knockdown to cytotoxicity by hydrogen peroxide, we assayed cytotoxicity by hydrogen peroxide with PERK knockdown in the presence of NAC. When NAC was added to hydrogen peroxide with PERK knockdown, the increased cytotoxicity of hydrogen peroxide with PERK knockdown was abolished in both doxorubicin-resistant cancer cell lines (Fig. 5B).

When doxorubicin was used as an agent exhibiting oxidative stress instead of hydrogen peroxide, cytotoxicity to doxorubicin with PERK knockdown in doxorubicin-resistant cancer cell lines increased, although an increase of cytotoxicity by PERK knockdown was modest (Fig. 5C). In addition, NAC addition abolished this favorable effect of 
PERK knockdown similar to when hydrogen peroxide was used (Fig. 5D).

\section{Discussion}

Sorafenib exerts anti-tumor effects through inhibition of multiple kinases, such as Raf-1, VEGFR 2 and 3, PDGFR-B, FLT-3, c-KIT and RET-receptor tyrosine kinase $(16,17)$. However, it remains unknown what target sorafenib acts on during cell cycle progression to inhibit cell proliferation. In this study, cell cycle analysis revealed that sorafenib induced cell cycle arrest at G2 phase, leading to cellular apoptosis in doxorubicin-resistant cancer cells. Cell cycle proceedings are controlled by modification of players involved in cell cycle progression such as phosphorylation and acetylation $(34,35)$. Therefore, sorafenib activity could involve inhibition of kinases involved in mitotic process. There are many players which work on mitosis such as polo-like kinase (36), aurora kinase (37) and topoisomerase (38). In this study, cell cycle arrest at G2 phase was more prominent by sorafenib treatment compared with PERK knockdown. This discrepancy of cell cycle analysis between sorafenib treatment and PERK knockdown indicated that some of kinases involved in mitosis progression in addition to PERK might be responsible for cell cycle arrest at G2 phase by sorafenib treatment.

Pancreatic $B$-islet cells produce large amounts of proinsulin proteins and are continuously exposed to ER stress leading to cellular apoptosis. Therefore, pancreatic $\beta$-islet cells regulate protein synthesis through a control of translation level. In this process of translation control, PERK plays a critical role to phosphorylate eIF $2 \alpha$ and repress translation except for ATF4. Mutation in PERK was shown to induce diabetes mellitus through pancreatic $\beta$-islet cells apoptosis, probably due to disorder of a mechanism of unfolded protein reaction (UPR) (39). Sorafenib has also been associated with pancreatic disorder and drug-induced pancreatitis which occurs during sorafenib administration, suggesting that multiple kinase inhibitor, sorafenib could inhibit PERK function (40). Results clearly showed that phosphorylation of eIF $2 \alpha$ was reduced by sorafenib treatment (Fig. 2B). There are recognized to be four eIF2 $\alpha$ kinases, that is, HRI, PRK, PERK and GCN2. In urothelial cancer cells, three eIF2 $\alpha$ kinases except HRI are expected to be expressed. In KK47/ADR and T24/ADR cells, PERK seems to be responsible for phosphorylation of eIF2 $\alpha$ because only PERK knockdown could repress phosphorylation of eIF2 $\alpha$ as shown in Fig. 2C. Therefore, sorafenib is suggested to reduce phosphorylation of eIF $2 \alpha$ through an inhibition of PERK kinase activity, at least in KK47/ADR and T24/ADR cells. In addition, phosphorylation level of eIF $2 \alpha$ seemed to correlate with cellular sensitivity to sorafenib. Survival of doxorubicin-resistant cancer cells having high phosphorylation level of eIF $2 \alpha$ was suppressed more effectively by sorafenib and PERK knockdown. Similar findings that sorafenib reduced eIF $2 \alpha$ phosphorylation through PERK inhibition have been found also in RCC cells (32).

It is important task to improve the outcome of metastatic or recurrent solid tumor patients. Although so far, conventional chemotherapy using various anticancer agents such as cisplatin, doxorubicin and paclitaxel have been employed for the treatment of metastatic or recurrent solid tumors, results are not satisfactory. Furthermore, there is no effective therapy when solid tumors become resistant to conventional chemotherapy. Recently, new small molecule agents were administered for metastatic RCC patients, and one of them is sorafenib. Sorafenib in combination with doxorubicin has been reported to double the median overall survival from 6.5 months in the doxorubicin-mono therapy group to 13.7 months in the combination group in a randomized, doubleblind, placebo-controlled phase II study of patients with $\mathrm{HCC}$, indicating a possibility that sorafenib is effective in combination with doxorubicin (41). However, sorafenib in combination with conventional chemotherapeutic agents, paclitaxel plus carboplatin for melanoma patients failed to show improvement in overall survival (42). In this study, sorafenib in vitro could sensitize doxorubicin-resistant cancer cell lines possessing high eIF $2 \alpha$ phosphorylation status to oxidative stress exerted by hydrogen peroxide and doxorubicin, indicating that combination therapy of anticancer agents exerting oxidative stress and sorafenib may be a beneficial therapeutic approach for cancer cells dependent on eIF $2 \alpha$ pathway. In addition, doxorubicin-resistant cancer cells were more sensitive to sorafenib than their parental cells, suggesting that sorafenib might be very useful after progression in the chemotherapy using doxorubicin-containing regimens. However, further investigation of the effect of combination therapeutics of sorafenib and doxorubicin are needed using in vivo models and clinical studies. In addition, the PERK silencing and other modulators of PERK activity with or without doxorubicin would also be beneficial for doxorubicin-resistant cancer cells.

In conclusion, the multikinase inhibitor sorafenib was found to inhibit the eIF $2 \alpha$ pathway regulating protein translation. Silencing of PERK sensitized doxorubicinresistant cells to oxidative stress by hydrogen peroxide and doxorubicin. In addition, sorafenib sensitized to hydrogen peroxide and doxorubicin. These findings indicate that sorafenib might be a useful agent for treatment of solid tumors in combination with doxorubicin in progressive cancer after chemotherapy using doxorubicin-containing regimens.

\section{Acknowledgements}

This work was in part supported by Health Sciences Research Grants for Clinical Research for Evidenced Based Medicine and Grants-in-Aid for Cancer Research (016), from the Ministry of Health, Labor and Welfare, Japan. We would like to thank Dr Dongchon Kang (Kyushu University, Fukuoka, Japan) for helping with flow cytometry, and Noriko Hakoda and Seiko Kamori for their technical assistance.

\section{References}

1. Kotoh S, Naito S, Yokomizo A, et al: Increased expression of DNA topoisomerase I gene and collateral sensitivity to camptothecin in human cisplatin-resistant bladder cancer cells. Cancer Res 54: 3248-3252, 1994.

2. Kotoh S, Naito S, Yokomizo A, Kohno K, Kuwano M and Kumazawa J: Enhanced expression of gamma-glutamylcysteine synthetase and glutathione S-transferase genes in cisplatinresistant bladder cancer cells with multidrug resistance phenotype. J Urol 157: 1054-1058, 1997. 
3. Yokomizo A, Ono M, Nanri H, et al: Cellular levels of thioredoxin associated with drug sensitivity to cisplatin, mitomycin C, doxorubicin, and etoposide. Cancer Res 55: 4293-4296, 1995.

4. Hasegawa S, Abe T, Naito S, et al: Expression of multidrug resistance-associated protein (MRP), MDR1 and DNA topoisomerase II in human multidrug-resistant bladder cancer cell lines. Br J Cancer 71: 907-913, 1995.

5. Tada Y, Wada M, Kuroiwa K, et al: MDR1 gene overexpression and altered degree of methylation at the promoter region in bladder cancer during chemotherapeutic treatment Clin Cancer Res 6: 4618-4627, 2000

6. Tada Y, Wada M, Migita T, et al: Increased expression of multidrug resistance-associated proteins in bladder cancer during clinical course and drug resistance to doxorubicin. Int J Cancer 98: 630-635, 2002.

7. Tsunoda T, Koga H, Yokomizo A, et al: Inositol 1,4,5-trisphosphate (IP3) receptor type1 (IP3R1) modulates the acquisition of cisplatin resistance in bladder cancer cell lines. Oncogene 24 1396-1402, 2005.

8. Yasui K, Mihara S, Zhao C, et al: Alteration in copy numbers of genes as a mechanism for acquired drug resistance. Cancer Res 64: 1403-1410, 2004.

9. Miyamoto $\mathrm{N}$, Izumi $\mathrm{H}$, Noguchi $\mathrm{T}$, et al: Tip60 is regulated by circadian transcription factor clock and is involved in cisplatin resistance. J Biol Chem 283: 18218-18226, 2008

10. Shiota M, Izumi H, Onitsuka T, et al: Twist promotes tumor cell growth through YB-1 expression. Cancer Res 68: 98-105, 2008.

11. Shiota M, Izumi H, Tanimoto A, et al: Programmed cell death protein 4 down-regulates $\mathrm{Y}$-box binding protein-1 expression via a direct interaction with Twist 1 to suppress cancer cell growth. Cancer Res 69: 3148-3156, 2009.

12. Naito S, Yokomizo A and Koga H: Mechanisms of drug resistance in chemotherapy for urogenital carcinoma. Int J Urol 6: 427-439, 1999.

13. Naito S, Koga H, Yokomizo A, et al: Molecular analysis of mechanisms regulating drug sensitivity and the development of new chemotherapy strategies for genitourinary carcinomas. World J Surg 24: 1183-1186, 2000.

14. Wilhelm S and Chien DS: BAY 43-9006: preclinical data. Curr Pharm Des 8: 2255-2257, 2002.

15. Lyons JF, Wilhelm S, Hibner B and Bollag G: Discovery of a novel Raf kinase inhibitor. Endocr Relat Cancer 8: 219-225, 2001.

16. Wilhelm SM, Carter C, Tang L, et al: BAY 43-9006 exhibits broad spectrum oral antitumor activity and targets the RAF/ MEK/ERK pathway and receptor tyrosine kinases involved in tumor progression and angiogenesis. Cancer Res 64: 7099-7109, 2004.

17. Eto $\mathrm{M}$ and Naito $\mathrm{S}$ : Molecular targeting therapy for renal cell carcinoma. Int J Clin Oncol 11: 209-213, 2006.

18. Abou-Alfa GK, Schwartz L, Ricci S, et al: Phase II study of sorafenib in patients with advanced hepatocellular carcinoma. J Clin Oncol 24: 4293-4300, 2006.

19. Escudier B, Eisen T, Stadler WM, et al: Sorafenib in advanced clear-cell renal-cell carcinoma. N Engl J Med 356: 125-134, 2007.

20. Akaza H, Tsukamoto T, Murai M, Nakajima $\mathrm{K}$ and Naito S: Phase II study to investigate the efficacy, safety, and pharmacokinetics of sorafenib in Japanese patients with advanced renal cell carcinoma. Jpn J Clin Oncol 37: 755-762, 2007.

21. Dever TE: Gene-specific regulation by general translation factors. Cell 108: 545-556, 2002.

22. Holcik M and Sonenberg N: Translational control in stress and apoptosis. Nat Rev Mol Cell Biol 6: 318-327, 2005.
23. Wek RC, Jiang HY and Anthony TG: Coping with stress: eIF2 kinases and translational control. Biochem Soc Trans 34: 7-11, 2006.

24. Ron D and Walter P: Signal integration in the endoplasmic reticulum unfolded protein response. Nat Rev Mol Cell Biol 8: 519-529, 2007.

25. Marciniak SJ and Ron D: Endoplasmic reticulum stress signaling in disease. Physiol Rev 86: 1133-1149, 2006.

26. Schröder M and Kaufman RJ: The mammalian unfolded protein response. Annu Rev Biochem 74: 739-789, 2005.

27. Harding HP, Zhang Y, Zeng H, et al: An integrated stress response regulates amino acid metabolism and resistance to oxidative stress. Mol Cell 11: 619-633, 2003.

28. Vattem KM and Wek RC: Reinitiation involving upstream ORFs regulates ATF4 mRNA translation in mammalian cells. Proc Natl Acad Sci USA 101: 11269-11274, 2004.

29. Lu PD, Harding HP and Ron D: Translation reinitiation at alternative open reading frames regulates gene expression in an integrated stress response. J Cell Biol 167: 27-33, 2004.

30. Shiota M, Izumi H, Onitsuka T, et al: Twist and $\mathrm{p} 53$ reciprocally regulate target genes via direct interaction. Oncogene 27: 5543-5553, 2008.

31. Shiota M, Yokomizo A, Tada Y, et al: Castration resistance of prostate cancer cells caused by castration-induced oxidative stress through Twist 1 and androgen receptor overexpression. Oncogene 29: 237-250, 2010.

32. Shiota M, Eto M, Yokomizo A, et al: Sorafenib with doxorubicin augments cytotoxicity to renal cell cancer through PERK inhibition. Int J Oncol 36: 1521-1531, 2010.

33. Benchekroun MN, Catroux P, Montaudon D and Robert J: Development of mechanisms of protection against oxidative stress in doxorubicin-resistant rat tumoral cells in culture. Free Radic Res Commun 11: 137-144, 1990.

34. Reinhardt HC and Yaffe MB: Kinases that control the cell cycle in response to DNA damage: Chk1, Chk2, and MK2. Curr Opin Cell Biol 21: 245-255, 2009.

35. McManus KJ and Hendzel MJ: The relationship between histone $\mathrm{H} 3$ phosphorylation and acetylation throughout the mammalian cell cycle. Biochem Cell Biol 84: 640-657, 2006.

36. Archambault V and Glover DM: Polo-like kinases: conservation and divergence in their functions and regulation. Nat Rev Mol Cell Biol 10: 265-275, 2009

37. Vader G and Lens SM: The Aurora kinase family in cell division and cancer. Biochim Biophys Acta 1786: 60-72, 2008.

38. Nitiss JL: DNA topoisomerase II and its growing repertoire of biological functions. Nat Rev Cancer 9: 327-337, 2009.

39. Shi Y, An J, Liang J, et al: Characterization of a mutant pancreatic eIF-2alpha kinase, PEK, and co-localization with somatostatin in islet delta cells. J Biol Chem 274: 5723-5730, 1999.

40. Kane RC, Farrell AT, Saber H, et al: Sorafenib for the treatment of advanced renal cell carcinoma. Clin Cancer Res 12: 7271-7278, 2006.

41. Richly H, Schultheis B, Adamietz IA, et al: Combination of sorafenib and doxorubicin in patients with advanced hepatocellular carcinoma: results from a phase I extension trial. Eur J Cancer 45: 579-587, 2009.

42. Hauschild A, Agarwala SS, Trefzer U, et al: Results of a phase III, randomized, placebo-controlled study of sorafenib in combination with carboplatin and paclitaxel as second-line treatment in patients with unresectable stage III or stage IV melanoma. J Clin Oncol 27: 2823-2830, 2009. 